\title{
AN ANALOGUE OF THE KOLOSOFF-MUSKHELISHVILI FORMULAE IN THREE DIMENSIONS*
}

\author{
BY \\ DARRELL D. PENROD \\ University of Notre Dame, Notre Dame, Indiana
}

1. Introduction. The application of analytic function theory to two dimensional elasticity has contributed greatly to the development of that area. While there is no complete generalization of this technique for three dimensions, there is, in fact, a theory of functions of a hypervariable which can be utilized. The hypervariable is that of Ketchum [1], and monogenic functions of this hypervariable generate harmonic functions in three dimensions. Since the displacement vector of a linear isotropic elastic solid can be represented in terms of harmonic functions, it can also be represented in terms of functions of the hypervariable. This representation is accomplished herein and used to generate several singular solutions for the infinite and semi-infinite elastic space.

2. Hypernumbers and functions of the hypervariables. A hypernumber $B$ has the following form:

$$
B=\sum_{k=-\infty}^{\infty} b_{k} E_{k}
$$

where each $b_{k}$ is a complex number and $E_{k}$ is a basis element for the algebra. The ordinary algebraic operations for hypernumbers are defined below. If $B=\sum_{k=-\infty}^{\infty} b_{k} E_{k}$ and $C=\sum_{k=-\infty}^{\infty} c_{k} E_{k}$ then

$$
\begin{gathered}
B \pm C=\sum_{k=-\infty}^{\infty}\left(b_{k} \pm c_{k}\right) E_{k}, \quad \lambda B=\sum_{k=-\infty}^{\infty}\left(\lambda b_{k}\right) E_{k} \\
B C=\sum_{k=-\infty}^{\infty} d_{k} E_{k} \quad \text { where } \quad d_{k}=\sum_{i=-\infty}^{\infty} b_{k-j} c_{i}
\end{gathered}
$$

provided $d_{k}$ converges for each $k$. If one or more $d_{k}$ is infinite, then the product is not defined.

We shall be concerned with functions of the hypervariable

$$
w=-v E_{-1}+x E_{0}+u E_{1}
$$

where

$$
u=\frac{1}{2}(z+i y) \text { and } v=\frac{1}{2}(z-i y)
$$

A hyperfunction

$$
F(x, u, v)=\sum_{k=-\infty}^{\infty} f_{k}(x, u, v) E_{k}
$$

*Received January 18, 1965; Revised manuscript received April 16, 1965. Portions of this paper are based on the author's doctoral dissertation completed at the University of Illinois, 1964. 
is said to have a derivative with respect to $w$ if its component functions $f_{k}$ satisfy the following equations

$$
-\frac{\partial f_{k-1}}{\partial v}=\frac{\partial f_{k}}{\partial x}=\frac{\partial f_{k+1}}{\partial u} \quad(k=0, \pm 1, \pm 2 \cdots)
$$

When the derivative exists, it can be shown (Ketchum [1]) that

$$
\frac{d F}{d w}=\sum_{k=-\infty}^{\infty} \frac{\partial f_{k}}{\partial x} E_{k}
$$

Furthermore, when $d F / d w$ exists, and when the second partial derivatives of each $f_{k}$ exist and are continuous, we can differentiate (2.1) with respect to $x$ obtaining

$$
\frac{\partial^{2} f_{k}}{\partial x^{2}}=\frac{\partial^{2} f_{k+1}}{\partial x \partial u}
$$

Under the above assumptions

$$
\frac{\partial^{2} f_{k+1}}{\partial x \partial u}=\frac{\partial^{2} f_{k+1}}{\partial u \partial x}=\frac{\partial}{\partial u}\left(\frac{\partial f_{k+1}}{\partial x}\right) .
$$

But according to (2.1)

$$
\frac{\partial f_{k+1}}{\partial x}=-\frac{\partial f_{k}}{\partial v},
$$

and so

$$
\frac{\partial^{2} f_{k}}{\partial x^{2}}=\frac{\partial^{2} f_{k+1}}{\partial x \partial u}=\frac{\partial^{2} f_{k+1}}{\partial u \partial x}=\frac{\partial}{\partial u}\left(-\frac{\partial f_{k}}{\partial v}\right) .
$$

Recalling that $u=\frac{1}{2}(z+i y)$ and $v=\frac{1}{2}(z-i y)$ we have

$$
\frac{\partial^{2} f_{k}}{\partial x^{2}}+\frac{\partial^{2} f_{k}}{\partial y^{2}}+\frac{\partial^{2} f_{k}}{\partial z^{2}}=0 .
$$

A function which has a derivative for all values of $w$ in a region $D$, and whose component functions are analytic in $D$ is said to be monogenic in $D$.

The preceding discussion shows that the component functions of a monogenic function of $w$ are themselves harmonic. A partial converse to this result was given by Ketchum [1].

Theorem 1 (Completeness Theorem). If $g(x, y, z)$ is harmonic in a region $D$, then there exists a function $F(w)=\sum_{-\infty}^{\infty} f_{k} E_{k}$, monogenic in a subregion of $D$, with the property that $g(x, y, z)=f_{0}(x, u, v)$.

3. Displacements and stresses in terms of functions of the hypervariable. The desired hyperfunction representation is based upon a harmonic representation deduced from a result due to Duffin [2].

Lemma 1. If $g(x, y, z)$ is a harmonic function in the cylindrical region $C$ defined by $y^{2}+z^{2} \leq a^{2}$ and $0 \leq x \leq b$, then in a cylinder $C_{1} \subset C$ there exists a harmonic function $h(x, y, z)$ such that

$$
\frac{\partial h}{\partial x}=g
$$


Theorem 2. If $\mathbf{u}$ is a vector valued function of class $C^{3}$ in a cylinder $C$, and if $\mathbf{u}$ is a solution of the homogeneous Cauchy-Navier equation in $C$, then there exist functions $f, g$, and $h$, harmonic in $C_{1} \subset C$ such that

$$
\mathbf{u}=-\nabla \times g \mathbf{i}+\frac{\lambda+3 \mu}{2 \mu} f \mathbf{i}-\frac{\lambda+\mu}{2 \mu} x \nabla f-\nabla h
$$

in $C_{1}$.

Proof: The Cauchy-Navier equation is

$$
\frac{\lambda+2 \mu}{\mu} \nabla(\nabla \cdot \mathbf{u})-\nabla \times \nabla \times \mathbf{u}=0
$$

where $\lambda$ and $\mu$ are constants (nonzero). Since the components of $\mathbf{u}$ are of class $C^{3}$, the divergence of the above equation exists and is

$$
\frac{\lambda+2 \mu}{\mu} \nabla^{2}(\nabla \cdot \mathfrak{u})=0 .
$$

Hence we put $\nabla \cdot \mathbf{u}=\partial f / \partial x$ where $f$ is harmonic, (lemma 1 ). We also notice that since $f$ is harmonic,

$$
\nabla(\nabla \cdot \mathfrak{u})=\nabla \frac{\partial f}{\partial x}=\nabla \times \nabla \times f \mathbf{i}
$$

and (2.3) becomes

$$
\nabla \times \nabla \times\left[\frac{\lambda+2 \mu}{\mu} f \mathbf{i}-\mathbf{u}\right]=0
$$

It follows that

and

$$
\nabla \times\left[\frac{\lambda+2 \mu}{\mu} f \mathbf{i}-\mathfrak{u}\right]=\nabla \varphi
$$

$$
\nabla^{2} \varphi=0 .
$$

Let $\varphi=\partial g / \partial x$ where $g$ is harmonic (lemma 1), and again $\nabla \partial g / \partial x=\nabla \times \nabla \times g \mathbf{i}$. Then equation (2.4) is

$$
\nabla \times\left[\frac{\lambda+2 \mu}{\mu} f \mathbf{i}-\mathfrak{u}-\nabla \times g \mathbf{i}\right]=0
$$

which implies that

$$
\frac{\lambda+2 \mu}{\mu} f \mathbf{i}-\mathbf{u}-\nabla \times g \mathbf{i}=\nabla \psi
$$

Taking the divergence of equation (2.5) and recalling that $\nabla \cdot \mathfrak{u}=\partial f / \partial x$, we have

$$
\frac{\lambda+\mu}{\mu} \frac{\partial f}{\partial x}=\nabla^{2} \psi
$$

However, $f$ is harmonic so that

$$
\nabla^{2}\left(\psi-\frac{\lambda+\mu}{2 \mu} x f\right)=0
$$


hence

$$
\psi=\frac{\lambda+\mu}{2 \mu} x f+h
$$

where $h$ is harmonic. Substitution into equation (2.5) yields the desired result.

The scalar form of $(2.2)$ is

$$
\begin{aligned}
& u_{1}=\frac{\lambda+3 \mu}{2 \mu} f-\frac{\lambda+\mu}{2 \mu} x \frac{\partial f}{\partial x}-\frac{\partial h}{\partial x} \\
& u_{2}=-\frac{\partial g}{\partial z}-\frac{\lambda+\mu}{2 \mu} x \frac{\partial f}{\partial y}-\frac{\partial h}{\partial y} \\
& u_{3}=\frac{\partial g}{\partial y}-\frac{\lambda+\mu}{2 \mu} x \frac{\partial f}{\partial z}-\frac{\partial h}{\partial z}
\end{aligned}
$$

Let $P(w)=\sum_{k--\infty}^{\infty} p_{k} E_{k}$ be a monogenic function of $w$, then $P^{i}$ will denote the $j$-th component function of $P(w)$, that is,

$$
P^{i}=p_{i}
$$

Theorem 3. If $\mathbf{u}$ is a solution of the homogeneous Cauchy-Navier equation and of class $C^{3}$ in a region $D$, then, in some subregion of $D$, there exist monogenic functions $F(w), G(w)$ and $H(w)$ such that

$$
\begin{aligned}
u_{1} & =\frac{\lambda+3 \mu}{2 \mu} F^{0}-\frac{\lambda+\mu}{2 \mu} x \frac{d F^{0}}{d w}-\frac{d H^{0}}{d w} \\
u_{2}-i u_{3} & =\frac{d G^{1}}{d w}-i \frac{\lambda+\mu}{2 \mu} x \frac{d F^{1}}{d w}-i \frac{d H^{1}}{d w} \\
u_{2}+i u_{3} & =-\frac{d G^{-1}}{d w}-i \frac{\lambda+\mu}{2 \mu} x \frac{d F^{-1}}{d w}-i \frac{d H^{-1}}{d w} .
\end{aligned}
$$

Proof: It has already been shown that

$$
\begin{aligned}
& u_{1}=\frac{\lambda+3 \mu}{2 \mu} f-\frac{\lambda+\mu}{2 \mu} x \frac{\partial f}{\partial x}-\frac{\partial h}{\partial x} \\
& u_{2}=-\frac{\partial g}{\partial z}-\frac{\lambda+\mu}{2 \mu} x \frac{\partial f}{\partial y}-\frac{\partial h}{\partial y} \\
& u_{3}=\frac{\partial g}{\partial y}-\frac{\lambda+\mu}{2 \mu} x \frac{\partial f}{\partial z}-\frac{\partial h}{\partial z}
\end{aligned}
$$

where $f, g$, and $h$ are harmonic. Let three monogenic functions $F, G, H$ be chosen (theorem 1) such that

Then

$$
F^{0}=f, \quad G^{0}=g, \quad H^{0}=h .
$$

$$
\begin{aligned}
& \frac{d F^{0}}{d w}=\frac{\partial f}{\partial x}, \quad \frac{d F^{1}}{d w}=-\frac{\partial f}{\partial v}=-i \frac{\partial f}{\partial y}-\frac{\partial f}{\partial z} \\
& \frac{d F^{-1}}{d w}=\frac{\partial f}{\partial u}=-i \frac{\partial f}{\partial y}+\frac{\partial f}{\partial z},
\end{aligned}
$$

and similarly for $G$ and $H$. 
Finally, we have

$$
\begin{gathered}
\frac{\lambda+3 \mu}{2 \mu} F^{0}-\frac{\lambda+\mu}{2 \mu} x \frac{d F^{0}}{d w}-\frac{d H^{0}}{d w}=\frac{\lambda+3 \mu}{2 \mu} f-\frac{\lambda+\mu}{2 \mu} x \frac{\partial f}{\partial x}-\frac{\partial h}{\partial x}=u_{1}, \\
\frac{d G^{1}}{d w}-i \frac{\lambda+\mu}{2 \mu} x \frac{d F^{1}}{d w}-i \frac{d H^{1}}{d w} \\
=\left(-\frac{\partial g}{\partial z}-\frac{\lambda+\mu}{2 \mu} x \frac{\partial f}{\partial y}-\frac{\partial h}{\partial y}\right)-i\left(\frac{\partial g}{\partial y}-\frac{\lambda+\mu}{2 \mu} x \frac{\partial f}{\partial z}-\frac{\partial h}{\partial z}\right)=u_{2}-i u_{3},
\end{gathered}
$$

and

$$
\begin{aligned}
& -\frac{d G^{-1}}{d w}-i \frac{\lambda+\mu}{2 \mu} x \frac{d F^{-1}}{d w}-i \frac{d H^{-1}}{d w} \\
& \quad=\left(-\frac{\partial g}{\partial z}-\frac{\lambda+\mu}{2 \mu} x \frac{\partial f}{\partial y}-\frac{\partial h}{\partial y}\right)+i\left(\frac{\partial g}{\partial y}-\frac{\lambda+\mu}{2 \mu} x \frac{\partial f}{\partial z}-\frac{\partial h}{\partial z}\right)=u_{2}+i u_{3}
\end{aligned}
$$

as required.

The corresponding equations for the stresses are derived from the following relations:

$$
\begin{gathered}
\mu\left[\frac{\partial u_{1}}{\partial v}+i \frac{\partial\left(u_{2}-i u_{3}\right)}{\partial x}\right]=\mu\left[\frac{\partial u_{1}}{\partial z}+i \frac{\partial u_{1}}{\partial y}+i \frac{\partial u_{2}}{\partial x}+\frac{\partial u_{3}}{\partial x}\right]=\tau_{x z}+i \tau_{x y}, \\
\mu \frac{\partial\left(u_{2}-i u_{3}\right)}{\partial v}=\mu\left[\frac{\partial u_{2}}{\partial z}+i \frac{\partial u_{2}}{\partial y}-i \frac{\partial u_{3}}{\partial z}+\frac{\partial u_{3}}{\partial y}\right]=\tau_{y s}+\frac{i\left(\sigma_{y}-\sigma_{z}\right)}{2}, \\
\nabla \cdot \mathbf{u}=\frac{d F^{0}}{d w}, \\
\lambda \nabla \cdot \mathbf{u}+2 \mu \frac{\partial u_{1}}{\partial x}=\sigma x .
\end{gathered}
$$

Utilizing equations (2.1) and (2.7) yields the stress hyperfunction relations

$$
\begin{gathered}
\sigma_{x}=(\lambda+2 \mu) \frac{d F^{0}}{d w}-(\lambda+\mu) x \frac{d^{2} F^{0}}{d w^{2}}-2 \mu \frac{d^{2} H^{0}}{d w^{2}} \\
\tau_{x z}+i \tau_{x y}=-\mu \frac{d F^{1}}{d w}+(\lambda+\mu) x \frac{d^{2} F^{1}}{d w^{2}}+2 \mu \frac{d^{2} H^{1}}{d w^{2}}+\mu i \frac{d^{2} G^{1}}{d w^{2}} \\
\tau_{y z}+\frac{i\left(\sigma_{y}-\sigma_{z}\right)}{2}=-\mu \frac{d^{2} G^{2}}{d w^{2}}+\frac{i(\lambda+\mu)}{2} x \frac{d^{2} F^{2}}{d w^{2}}+\mu i \frac{d^{2} H^{2}}{d w^{2}} \\
\sigma_{x}+\sigma_{y}+\sigma_{z}=(3 \lambda+2 \mu) \frac{d F^{0}}{d w} .
\end{gathered}
$$

4. Applications. Many of the important solutions in three dimensional elasticity involve displacements of order $(1 / \rho)\left(\rho^{2}=x^{2}+y^{2}+z^{2}\right)$, and it is quite natural to seek monogenic functions of the same order. Knerr [3] has given a function $S(w)$ of the desired type. Let

$$
S(w)=\sum_{-\infty}^{\infty} s_{n} E_{n}
$$


where

$$
\begin{aligned}
s_{0} & =\frac{1}{\rho} \\
s_{n} & =\frac{(-1)^{n}(z+i y)^{n}}{\rho(\rho+x)^{n}} \quad(n=0,1,2, \cdots) \\
s_{-n} & =\frac{(z-i y)^{n}}{\rho(\rho+x)^{n}}
\end{aligned}
$$

where

$$
\rho^{2}=x^{2}+y^{2}+z^{2} \text {. }
$$

It is easily verified that this function is monogenic except along the negative $x$ axis and at the origin where it ceases to exist.

A number of solutions are generated by direct substitution into the displacementhyperfunction relations. These solutions have either point or line singularities and are called nuclei of strain by Love [4]. The use of the function $S(w)$ in the displacementhyperfunction relations (2.7) is illustrated below, and the various nuclei of strain are identified.

1. Let $F(w)=S(w), G(w)=H(w)=0$. Then

$$
\begin{aligned}
& u_{1}=\frac{\lambda+3 \mu}{2 \mu} \frac{1}{\rho}+\frac{\lambda+\mu}{2 \mu} \frac{x^{2}}{\rho^{3}} \\
& u_{2}=\frac{\lambda+\mu}{2 \mu} \frac{x y}{\rho^{3}} \\
& u_{3}=\frac{\lambda+\mu}{2 \mu} \frac{x z}{\rho^{3}} .
\end{aligned}
$$

(Point load in the $x$ direction at the origin)

2 .

$$
\begin{aligned}
F(w) & =G(w)=0, \quad H(w)=S(w) ; \\
u_{1} & =x / \rho^{3}, \quad u_{2}=y / \rho^{3}, \quad u_{3}=z / \rho^{3}
\end{aligned}
$$

(center of dilatation at the origin)

3.

$$
\begin{aligned}
F(w) & =H(w)=0, \quad G(w)=S(w) ; \\
u_{1} & =0, \quad u_{2}=z / \rho^{3}, \quad u_{3}=-y / \rho^{3}
\end{aligned}
$$

(Center of rotation about the $x$ axis at the origin)

4.

$$
\begin{aligned}
F(w) & =G(w)=0, \quad \frac{d H}{d w}=-S(w) ; \\
u_{1} & =\frac{1}{\rho}, \quad u_{2}=\frac{y}{\rho(\rho+x)}, \quad u_{3}=\frac{z}{\rho(\rho+x)}
\end{aligned}
$$

(Line of centers of dilatation along the axis from $x=0$ to $x=-\infty$ )

5.

$$
F(w)=H(w)=0, \quad \frac{d G}{d w}=S(w) ;
$$




$$
u_{1}=0, \quad u_{2}=\frac{-z}{\rho(\rho+x)}, \quad u_{3}=\frac{y}{\rho(\rho+x)}
$$

(Line of centers of rotation about the $x$ axis from $x=0$ to $x=-\infty$ )

6.

$$
\begin{aligned}
F(w) & =S(w), \quad G(w)=0, \quad \frac{d H}{d w}=\frac{1}{2} S(w) ; \\
u_{1} & =\frac{\lambda+\mu}{2 \mu} \frac{1}{\rho}+\frac{\lambda+\mu}{2 \mu} \frac{x^{2}}{\rho^{3}}, \\
u_{2} & =\frac{\lambda+\mu}{2 \mu} \frac{x y}{\rho^{3}}-\frac{y}{\rho(\rho+x)}, \\
u_{3} & =\frac{\lambda+\mu}{2 \mu} \frac{x z}{\rho^{3}}-\frac{z}{\rho(\rho+x)}
\end{aligned}
$$

(Point load at the origin normal to the half space $x \geq 0$ with no other surface tractions on $x=0$; Boussinesq [5])

7.

$$
\begin{aligned}
F & =\frac{1}{2(\lambda+\mu)}\left(E_{1}-E_{-1}\right) S(w), \quad \frac{d G}{d w}=\frac{-i}{2 \mu}\left(E_{1}+E_{-1}\right) S(w), \\
\frac{d H}{d w} & =\frac{\lambda+2 \mu}{4 \mu(\lambda+\mu)}\left(E_{1}-E_{-1}\right) S(w) ; \\
u_{1} & =\frac{1}{2(\lambda+\mu)} \frac{z}{\rho(\rho+x)}+\frac{1}{2 \mu} \frac{x z}{\rho^{3}}, \\
u_{2} & =\frac{1}{2 \mu} \frac{y z}{\rho^{3}}-\frac{1}{2(\lambda+\mu)} \frac{y z}{\rho(\rho+x)^{2}}, \\
u_{3} & =\frac{1}{2 \mu}\left(\frac{1}{\rho}+\frac{z^{2}}{\rho^{3}}\right)+\frac{1}{2(\lambda+\mu)}\left[\frac{1}{\rho+x}-\frac{z^{2}}{\rho(\rho+x)^{2}}\right]
\end{aligned}
$$

(Point load at the origin in the $z$ direction on the half space $x \geq 0$ with no other surface tractions on $x=0$; Cerruti [6])

The generation of solutions with singularities interior to the half space $x \geq 0$ necessitates the introduction of two new monogenic functions. Let

$$
S^{\prime}(w)=\sum_{n=-\infty}^{\infty} s_{n}^{\prime} E_{n}
$$

where

$$
\begin{aligned}
s_{n}^{\prime} & =\frac{(-1)^{n}(z+i y)^{n}}{\rho^{\prime}\left(\rho^{\prime}+x-\xi\right)^{n}}, \quad n=0,1,2, \cdots \\
s_{-n}^{\prime} & =\frac{(z-i y)^{n}}{\rho^{\prime}\left(\rho^{\prime}+x-\xi\right)^{n}}, \\
\rho^{\prime 2} & =(x-\xi)^{2}+y^{2}+z^{2},
\end{aligned}
$$

and

$$
S^{\prime \prime}(w)=\sum_{n=-\infty}^{\infty} s_{n} E_{n}
$$


where

$$
\begin{aligned}
s_{n}^{\prime \prime} & =\frac{(-1)^{n}(z+i y)^{n}}{\rho^{\prime \prime}\left(\rho^{\prime \prime}+x+\xi\right)^{n}}, \\
s_{-n}^{\prime \prime} & =\frac{(z-i y)^{n}}{\rho\left(\rho^{\prime \prime}+x+\xi\right)^{n}}, \\
\rho^{\prime \prime 2} & =(x+\xi)^{2}+y^{2}+z^{2} .
\end{aligned}
$$

The following nuclei of strain have been obtained for the half space $x \geq 0$.

8.

$$
\begin{gathered}
F(w)=S^{\prime}(w)+\frac{\lambda+3 \mu}{\lambda+\mu} S^{\prime \prime}(w)-2 \xi \frac{d S^{\prime \prime}}{d w}, \quad G(w)=0, \\
\frac{d H}{d w}=-\frac{(\lambda+\mu) \xi}{2 \mu} \frac{d S^{\prime}}{d w}+\frac{\lambda+2 \mu}{\lambda+\mu} S^{\prime \prime}(w)-\frac{(\lambda+3 \mu) \xi}{\lambda+\mu} \frac{d S^{\prime \prime}}{d w}
\end{gathered}
$$

(A concentrated load in the $x$ direction at $(\xi, 0,0)$ with $\sigma_{x}=\tau_{x y}=\tau_{x z}=0$ on $x=0$; Mindlin [7]).

9.

$$
\begin{aligned}
F(w) & =S^{\prime}(w)-S^{\prime \prime}(w)+\frac{2 \xi(\lambda+\mu)}{\lambda+3 \mu} \frac{d S^{\prime \prime}}{d w} \\
\frac{d H}{d w} & =-\frac{(\lambda+\mu) \xi}{2 \mu} \frac{d S^{\prime}}{d w}+\frac{(\lambda+\mu) \xi}{2 \mu} \frac{d S^{\prime \prime}}{d w}, \\
G(w) & =0
\end{aligned}
$$

(A concentrated load in the $x$ direction at $(\xi, 0,0)$ with $u_{1}=u_{2}=u_{3}=0$ on $x=0$; Rongved [8]).

10.

$$
\begin{aligned}
F(w) & =S^{\prime}(w)-S^{\prime \prime}(w), \\
\frac{d H}{d w} & =-\frac{(\lambda+\mu) \xi}{2 \mu} \frac{d S^{\prime}}{d w}+\frac{(\lambda+\mu) \xi}{2 \mu} \frac{d S^{\prime \prime}}{d w}, \\
G(w) & =0
\end{aligned}
$$

(A concentrated load in the $x$ direction at $(\xi, 0,0)$ with $\sigma_{x}=u_{2}=u_{3}=0$ on $x=0$ ) 11.

$$
\begin{aligned}
F(w) & =S^{\prime}(w)-S^{\prime \prime}(w) \\
\frac{d H}{d w} & =-\frac{(\lambda+\mu) \xi}{2 \mu} \frac{d S^{\prime}}{d w}-\frac{(\lambda+\mu) \xi}{2 \mu} \frac{d S^{\prime \prime}}{d w} \\
G(w) & =0
\end{aligned}
$$

(A concentrated load in the $x$ direction at $(\xi, 0,0)$ with $u_{1}=\tau_{x y}=\tau_{x z}=0$ on $x=0$ ).

12. $F(w)=\frac{1}{2}\left[E_{1}-E_{-1}\right]\left[S^{\prime}(w)+\frac{\lambda+3 \mu}{\lambda+\mu} S^{\prime \prime}(w)+2 \xi \frac{d S^{\prime \prime}}{d w}\right]$

$$
\begin{aligned}
& \frac{d H}{d w}=\frac{1}{2}\left[E_{1}-E_{-1}\right]\left[\frac{\lambda+3 \mu}{2 \mu} S^{\prime}+\frac{\lambda^{2}+4 \mu \lambda+5 \mu^{2}}{2 \mu(\lambda+\mu)} S^{\prime \prime}\right. \\
& \left.\quad-\frac{(\lambda+\mu) \xi}{2 \mu} \frac{d S^{\prime}}{d w}+\frac{(\lambda+3 \mu) \xi}{2 \mu} \frac{d S^{\prime \prime}}{d w}\right] \\
& \frac{d G}{d w}=-\frac{(\lambda+2 \mu) i}{2 \mu}\left[E_{1}+E_{-1}\right]\left[S^{\prime}(w)+S^{\prime \prime}(w)\right]
\end{aligned}
$$


(A concentrated load in the $z$ direction at $(\xi, 0,0)$ with $\sigma_{x}=\tau_{x y}=\tau_{x z}=0$ on $x=0$; Mindlin [7])

13. $\quad F(w)=\frac{1}{2}\left[E_{1}-E_{-1}\right]\left[S^{\prime}(w)-S^{\prime \prime}(w)-\frac{2(\lambda+\mu) \xi}{\lambda+3 \mu} \frac{d S^{\prime \prime}}{d w}\right]$

$$
\begin{aligned}
& \frac{d H}{d w}=\frac{1}{2}\left[E_{1}-E_{-1}\right]\left[\frac{\lambda+3 \mu}{2 \mu} S^{\prime}(w)\right. \\
& \left.\quad-\frac{(\lambda+\mu) \xi}{2 \mu} \frac{d S^{\prime}}{d w}-\frac{\lambda+3 \mu}{2 \mu} S^{\prime \prime}(w)-\frac{(\lambda+\mu) \xi}{2 \mu} \frac{d S^{\prime \prime}}{d w}\right] \\
& \frac{d G}{d w}=-\frac{i(\lambda+2 \mu)}{2 \mu}\left[E_{1}+E_{-1}\right]\left[S^{\prime}(w)-S^{\prime \prime}(w)\right]
\end{aligned}
$$

(A concentrated load in the $z$ direction at $(\xi, 0,0)$ with $u_{1}=u_{2}=u_{3}=0$ on $x=0$; Rongved [8])

14. $\quad F(w)=\frac{1}{2}\left[E_{1}-E_{-1}\right]\left[S^{\prime}+S^{\prime \prime}\right]$

$$
\begin{aligned}
& \frac{d H}{d w}=\frac{1}{2}\left[E_{1}-E_{-1}\right]\left[\frac{\lambda+3 \mu}{2 \mu}\left(S^{\prime}(w)+S^{\prime \prime}(w)\right)-\frac{(\lambda+\mu) \xi}{2 \mu}\left(\frac{d S^{\prime}}{d w}-\frac{d S^{\prime \prime}}{d w}\right)\right] \\
& \frac{d G}{d w}=-\frac{(\lambda+2 \mu) i}{2 \mu}\left[E_{1}+E_{-1}\right]\left[S^{\prime}(w)+S^{\prime}(w)\right]
\end{aligned}
$$

(A concentrated load in the $z$ direction at $(\xi, 0,0)$ with $u_{1}=\tau_{x y}=\tau_{x z}=0$ on $x=0$ )

15. $\quad F(w)=\frac{1}{2}\left[E_{1}-E_{-1}\right]\left[S^{\prime}(w)-S^{\prime \prime}(w)\right]$

$$
\begin{aligned}
& \frac{d H}{d w}=\frac{1}{2}\left[E_{1}-E_{-1}\right]\left[\frac{\lambda+3 \mu}{2 \mu}\left(S^{\prime}(w)-S^{\prime \prime}(w)\right)-\frac{(\lambda+\mu) \xi}{2 \mu}\left(\frac{d S^{\prime}}{d w}+\frac{d S^{\prime \prime}}{d w}\right)\right] \\
& \frac{d G}{d w}=\frac{-i(\lambda+2 \mu)}{2 \mu}\left[E_{1}+E_{-1}\right]\left[S^{\prime}(w)-S^{\prime \prime}(w)\right]
\end{aligned}
$$

(A concentrated load in the $z$ direction at $(\xi, 0,0)$ with $\sigma_{x}=u_{2}=u_{3}=0$ on $x=0$ )

All of the preceding nuclei for the concentrated load in the $x$ direction were determined from the general expression

$$
\begin{aligned}
F(w) & =S^{\prime}(w)+A S^{\prime \prime}(w)+B \frac{d S^{\prime \prime}}{d w}, \\
\frac{d H}{d w} & =\frac{-(\lambda+\mu) \xi}{2 \mu} \frac{d S^{\prime}}{d w}+C S^{\prime \prime}(w)+D \frac{d S^{\prime \prime}}{d w}, \\
G(w) & =0
\end{aligned}
$$

where $A, B, C, D$ are real numbers adjusted to satisfy the boundary conditions. Similarly, the nuclei for the concentrated load in the $z$ direction are derived from the expression 


$$
\begin{aligned}
F(w) & =\frac{1}{2}\left[E_{1}-E_{-1}\right]\left[S^{\prime}(w)+A S^{\prime \prime}(w)+B \frac{d S^{\prime \prime}}{d w}\right], \\
\frac{d H}{d w} & =\frac{1}{2}\left[E_{1}-E_{-1}\right]\left[\frac{\lambda+3 \mu}{2 \mu} S^{\prime}-\frac{(\lambda+\mu) \xi}{2 \mu} \frac{d S^{\prime}}{d w}+C S^{\prime \prime}(w)+D \frac{d S^{\prime \prime}}{d w}\right], \\
\frac{d G}{d w} & =\frac{-i}{2}\left[E_{1}+E_{-1}\right]\left[\frac{\lambda+2 \mu}{\mu} S^{\prime}(w)+K S^{\prime \prime}(w)+L \frac{d S^{\prime \prime}}{d w}\right],
\end{aligned}
$$

where $A, B, C, D, K, L$ are real numbers.

Acknowledgment. The author wishes to express his gratitude to Professor Marvin C. Stippes, University of Illinois, for his many valuable suggestions concerning this paper.

\section{REFERENCES}

1. P. W. Ketchum, A Complete Solution of Laplace's Equation by an Infinite Hypervariable, American Journal of Mathematics, 51, 179-188 (1929)

2. R. J. Duffin, Analytic Continuation in Elasticity, Journal of Rat. Mech. and Anal., 5, 939-950 (1956)

3. H. W. Knerr, Functions of an Infinite Harmonic Hypervariable, A. B. Thesis, University of Illinois (1929)

4. A. E. H. Love, A Treatise on the Mathematical Theory of Elasticity, fourth edition, Dover Publications, New York, 1944

5. J. Boussinesq, Applications des Potentiels a l'Etude de l'Equilibre et du Mouvment des Solides Elastiques, Gauthier-Villars, Paris, 1885.

6. V. Cerruti, Ricerche intorno all' equilibrio de' corpi elastici isotropi, Acc. Lincei Mem. fis. mat., Roma (3) 13, 81-122 (1882)

7. R. D. Mindlin, Force at a Point in the Interior of a Semi-infinite Solid, Physics 7, 195-202 (1936)

8. L. Rongved, Force at a Point in the Interior of a Semi-infinite Solid with Fixed Boundary, J. A. M. 22, 545-546 (1955) 